\title{
Performance Comparison of Differential Space-time Signalling Schemes for OFDM Systems
}

\author{
W. Hashim*, F. Said*, B. Allen ${ }^{\dagger}$, A. H. Aghvami* and A.F. Ismail ${ }^{\ddagger}$ \\ ${ }^{*}$ Centre for Telecommunications Research, King's College London, 26-29 Drury Lane, \\ London WC2B 5RL, United Kingdom, E-mail: wahidah.hashim@kcl.ac.uk, fatin.said@kcl.ac.uk \\ †University of Oxford, United Kingdom, Email: Ben.Allen@iee.org \\ $\ddagger$ International Islamic University Malaysia, Malaysia, Email: af_ismail@iiu.edu.my
}

\begin{abstract}
Differential transmit diversity is an attractive alternative to its coherent counterpart, especially for multiple antenna systems where channel estimation is more difficult to attain compared to that of single antenna systems. In this paper we compare two different types of differential transmit diversity techniques for OFDM based transmissions. The first technique uses Differential Space-Time Block Codes (DSTBC) from orthogonal designs and the second uses the Differential Cyclic Delay Diversity (DCDD). The results compare the bit error performance for several transmit antenna configurations. The results show that DCDD offers a very close performance to that of DSTBC, with the advantage of a simplified receiver structure.
\end{abstract}

\section{INTRODUCTION}

Future mobile radio systems are expected to provide and serve a wide range of applications. Applications such as interactive video communication would require high data rates, and one promising technology to accomplish this is multi-carrier modulation such as the OFDM. OFDM has been known for its robustness to multipath propagation and its ease for utilising multiple antenna techniques [1]. The recent wireless broadband technology, WiMAX, has examined the potential of OFDM as part of the IEEE 802.16 standards. Multiple antenna techniques at the transmitter and receiver, on the other hand, can be applied to further increase the capacity and performance of such communication systems [2].

Several signalling schemes that support multiple antenna techniques have been studied, such as Bell Labs Layered Space Time (BLAST), which gives an extraordinarily bandwith efficiency. Another such method is space-time trellis code (STTC) which incorporates the Viterbi algorithm to decode the received signals. Hence possible code sequences are given by means of a trellis and associated coding gain. These codes have shown considerable performance gains for wireless communication at the expense of a rising decoding effort with increasing numbers of transmit antennas or trellis states [3]. Finally, space-time block codes (STBC) enable multiple transmitters and receivers to be used to achieve full transmit diversity, and utilises both the time and space domains. Achieving full diversity leads to a high spectral efficiency [2]. However this is only true for the two transmit antennas case for complex signal constellations. The bandwidth efficiency is reduced when higher numbers of transmit antennas are used. Unlike STTC, STBC do not provide a coding gain [9].

In addition, delay diversity (DD) [4], which has been applied to OFDM systems in [5], is another transmit diversity technique. It is called delay diversity because delayed replicas of the same information bearing signal are transmitted from several antennas and achieve full diversity with any number of antennas. Here, the signals are assigned specific delays in order to avoid any intersymbol interference. To this end, DD was extended to cyclic delay diversity (CDD) in [5] and [6] by employing cyclic delays instead of time delays to the signals without any restriction on number of transmit antennas. With cyclic delays the scheme introduces virtual channel echoes and thus increases the frequency selectivity of the channel. CDD requires an outer channel code in order to gain its advantage, however this does not detract from the spectral efficiency since most wireless system specify a channel code as part of the air interface. The difference between CDD and STBC lies at the receiver, where it requires no modification to the original structure. Thus CDD has a distinct advantage of having a comparatively simple transmit and receiver architecture. Table 1 shows the summary of these transmit diversity schemes in terms of their advantages and disadvantages.

All of the above mentioned techniques, however, require the transmission of independent pilot symbols from each antenna to provide channel estimates for coherent detection at the receiver. This reduces the spectral efficiency of the transmission as well as requiring associated processing, hence adding complexity at the receiver. A method of avoiding such drawbacks is to employ differential modulation techniques which requires no prior knowledge of the channel at the receiver [7]. Furthermore, in some situations, it is difficult to estimate the channel accurately enough to provide satisfactory performance. This is, for example, when the channel is changing rapidly.

In this paper we conduct a comparison between two differential transmit diversity schemes: DSTBC from orthogonal designs [8]; and DCDD [10] for a mobile radio system based on coded OFDM system. The paper is organised as follows, first we briefly explain DSTBC and DCDD. Secondly, the 
TABLE I

TRANSMIT DIVERSITY SCHEMES COMPARISONS

\begin{tabular}{|l|l|l|}
\hline $\begin{array}{l}\text { Transmit } \\
\text { Diversity Scheme }\end{array}$ & $\begin{array}{l}\text { Advantage } \\
\text { efficiency spectral } \\
\text { bps/Hz) }\end{array}$ & $\begin{array}{l}\text { Complexity } \\
\text { increases with } \\
\text { number of transmit } \\
\text { antennas }\end{array}$ \\
\hline BLAST & $\begin{array}{l}\text { Diversity gain and } \\
\text { coding gain }\end{array}$ & $\begin{array}{l}\text { Complexity of the } \\
\text { decoder increases } \\
\text { exponentially with } \\
\text { number of transmit } \\
\text { antennas }\end{array}$ \\
\hline STBC & $\begin{array}{l}\text { Orthogonality that } \\
\text { guaranteed linear } \\
\text { decoding }\end{array}$ & $\begin{array}{l}\text { No coding gain, } \\
\text { channel need to be } \\
\text { quasi-static }\end{array}$ \\
\hline DD & $\begin{array}{l}\text { Simple receiver } \\
\text { structure }\end{array}$ & $\begin{array}{l}\text { Limited to number } \\
\text { of transmit anten- } \\
\text { nas }\end{array}$ \\
\hline CDD & $\begin{array}{l}\text { Simple receiver } \\
\text { structure }\end{array}$ & $\begin{array}{l}\text { Require channel } \\
\text { code to show the } \\
\text { gain }\end{array}$ \\
\hline
\end{tabular}

channel model and the simulation system layout is described. Single antenna and uncoded DCDD and DSTBC OFDM systems have been added for comparison purposes.

\section{Differential Space-Time Block Codes and DifFERENTIAL CYCLIC DELAY DIVERSITY}

\section{A. Differential Space-Time Block Codes in OFDM}

In this section we briefly introduce DSTBC as reported in [8] and [11]. These codes are based on the theory of generalised orthogonal designs for real valued signal constellations. In this paper, only real signal constellations are considered following the Hiperlan $/ 2$ mode 1 system parameters. Furthermore, full rate code designs exist for the case of 2, 3 and 4 transmit antennas. In here, $N_{T}=2,3$ and 4 transmit antennas, are employed with $N_{R}=1$ receive antenna.

A DSTBC is defined by an orthogonal matrix, $\mathbf{X}_{\mathbf{N}_{\mathbf{T}}}$, of size $K \times N_{T}$ and provides a mapping rule for the BPSK transmit symbols $x_{1}$ to $x_{P}$. Here, $K$ is defined as the number of time slots in the transmission, and $P$ is the number of symbols taken in the codeword. The coding rate is calculated as rate $=$ $P / K$. The code matrix shown below is no different from the matrix used in a coherent STBC, however, each of the elements in the matrix now need to be differentially modulated before mapping onto the code matrix. For $N_{T}=2,3$ and 4, orthogonal designs are given below as described in [8] and [11].

$$
\begin{gathered}
\mathbf{X}_{2}=\left[\begin{array}{cc}
x_{1} & x_{2} \\
-x_{2} & x_{1}
\end{array}\right] \\
\mathbf{X}_{3}=\left[\begin{array}{cccc}
x_{1} & x_{2} & x_{3} & 0 \\
-x_{2} & x_{1} & 0 & x_{3} \\
-x_{3} & 0 & x_{1} & -x_{2} \\
0 & -x_{3} & x_{2} & x_{1}
\end{array}\right]
\end{gathered}
$$

$$
\mathbf{X}_{4}=\left[\begin{array}{cccc}
x_{1} & x_{2} & x_{3} & x_{4} \\
-x_{2} & x_{1} & -x_{4} & x_{3} \\
-x_{3} & x_{4} & x_{1} & -x_{2} \\
x_{4} & -x_{3} & x_{2} & x_{1}
\end{array}\right]
$$

The entries in the same row of $\mathbf{X}_{\mathbf{N}_{\mathbf{T}}}$ are transmitted simultaneously from different antennas, the entries in the same column are transmitted from the same antenna in successive time slots.

\section{Transmitter}

In [8], the calculation of coefficients are required for differentially encoding the STBC codeword $\mathbf{S}_{\mathbf{k}}$ prior to transmission. For 2 Tx-antennas with BPSK modulation, 4 coefficients are required. 16 coefficients are required for 3 and $4 \mathrm{Tx}$ antennas. Another method of differentially encoded the message is by using an approach similar to standard single-antenna DPSK [7]. The only difference is that, instead of a symbol as in DPSK, it is now in the form of a matrix. However this method is only applicable for a square matrix and with the orthogonality principle, both matrices will result in an identity matrix if they are multiplied with their own Hermitian form, $\mathbf{S}_{k} \mathbf{S}_{k}^{\dagger}=\mathbf{I}_{2}$. To initialise transmission, the transmitter sends a specified message codeword $\mathrm{X}_{0}$, which does not carry information. For simplicity, below is an example calculation for $2 \mathrm{Tx}$ antennas.

$$
\mathbf{X}_{k}=\mathbf{S}_{k} \mathbf{X}_{k-1}
$$

$\left[\begin{array}{rr}x_{1, k} & x_{2, k} \\ -x_{2, k} & x_{1, k}\end{array}\right]=\left[\begin{array}{cc}s_{1, k} & s_{2, k} \\ -s_{2, k} & s_{1, k}\end{array}\right]\left[\begin{array}{cc}x_{1, k-1} & x_{2, k-1} \\ -x_{2, k-1} & x_{1, k-1}\end{array}\right]$

The normalised power for this BPSK-modulated real vector is assumed to be $((1 / \sqrt{ } 2,0),(1 / \sqrt{ } 2,0)) .\left(s_{1, k}, s_{2, k}\right)$ can be any BPSK modulated symbol. The reader is referred to reference [15] for further details. For OFDM systems,

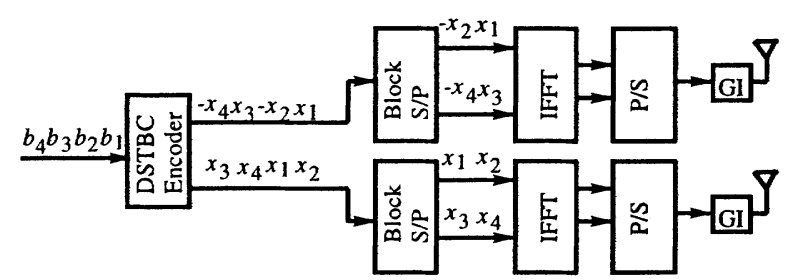

Fig. 1. Differential STBC OFDM Transmitter for 2 transmit antennas

the same encoding technique can be applied for $N_{T}=2$ with additional mapping of the transmitted symbols onto each subcarrier. Figure 1 depicts a simple example with $N_{T}=2$ and $N_{c}=2$ subcarriers using the DSTBC matrix, $\mathbf{X}_{2}$. This diagram does not include the channel encoder. $A$ guard interval, GI, is inserted before transmission to prevent intersymbol interference. 


\section{Receiver}

Under the assumption of constant channel coefficients during the transmission of $\mathbf{X}_{N_{T}}$, the orthogonal structure allows us to separate $x_{1} \ldots, x_{p}$ and to achieve the maximum possible level of diversity by linear combining. If the channel varies during the transmission of $\mathbf{X}_{N_{T}}$, the orthogonality would be lost, hence resulting in intersymbol interference.

For $1 \mathrm{Rx}$ antenna, after removing the guard interval and evaluating the FFT, the receive signal is described as shown in 4 , where $\eta_{t}$ are independent samples of a zero-mean complex Gaussian random variable with variance $1 /(2 \mathrm{SNR})$ per complex dimension and $\alpha_{n}$ is the path gain from each antenna.

$$
r_{t}=\sum_{n=1}^{N} \alpha_{n} X_{t}+\eta_{t}
$$

The decoding of DSTBC requires calculating the maximum likelihood (ML) detection for the estimated message $s_{k}$. Since differential decoding does not require for channel estimation, the detection is done by considering the previous receive signal, $r_{t, k-1}$ and the current receive signal, $r_{t, k}$, whereby $t$ represents the time slot. The same equation is used as with coherent detection, except that the channel estimate now is substituted with the previously conjugate receive symbols accordingly. For simplicity, the equation below is used to calculate the estimated $s_{1, k}$ and $s_{2, k}$ for the $2 \mathrm{Tx}$-antenna case.

$$
\begin{aligned}
s_{1, k} & =r_{1, k-1} r_{1, k}^{*}+r_{2, k}^{*} r_{2, k-1} \\
s_{2, k} & =r_{1, k-1} r_{2, k}^{*}-r_{2, k}^{*} r_{1, k-1}
\end{aligned}
$$

\section{B. Differential Cyclic Delay Diversity in OFDM}

\section{Transmitter}

At the transmitter, DCDD combines differential modulation, such as DPSK [7], with cyclic delay diversity as described in [10]. The output from the differential encoder is the difference between two adjacent input symbols, thus the transmitted symbol, $x(k)$, is the phase difference between the previously encoded symbol and the $k^{\text {th }}$ message symbol $s(k)$, as given by (6). The reference signal is assumed to be one.

$$
x(k)=s(k) x(k-1)
$$

CDD on the other hand, utilises the shifting of some symbols from the back of the OFDM block to the front, which is similar to adding the cyclic prefix in OFDM, where $\delta_{c y}$ is a 1 symbol delay.

Fig. 2 shows the DCDD transmitter architecture. The information bits are first channel encoded then modulated by a BPSK modulator. The channel encoder is important in the DCDD system since without the outer channel code, DCDD will not gain any advantage over a single antenna transmission. However, channel coding is considered common to many wireless systems. The modulated symbols are then differentially modulated and applied to the $N_{T}$ transmit antennas at the front end of the IFFT blocks. Each pair of antennas are treated separately. For each pair, the data stream is cyclically shifted, i.e., delayed by an antenna specific delay $\delta_{c y, l}$, where $l=$ $0,1,2 \ldots N_{T}$. The delay on the first antenna, in each set $\delta_{c y, 0}$, is zero. The choice of cyclic delays on other antennas have been investigated in [12] and [13] for OFDM systems. In [13] it is claimed that the best choice of cyclic delay for each antenna set can therefore be achieved by the following equation.

$$
\delta_{c y, l}=G+1+\delta_{c y, l-1}
$$

where $G$ is the guard interval. For $N_{T}=4$, the cyclic delays on the $2 \mathrm{nd}, 3 \mathrm{rd}$ and 4 th antennas are taken to be 17, 34 and 51 symbols respectively. These delays have been used in our simulation. In [?], the cyclic delays are calculated as follows.

$$
\delta_{c y, l}=\frac{N_{c}}{M_{B P S K} \times\left(2^{l-1}\right)} l=1,2, \ldots N_{T-1}
$$

where $M_{B P S K}$ is the modulation index. For $N_{T}=4, N_{c}=64$ subcarriers, the cyclic delays on the $2 \mathrm{nd}, 3 \mathrm{rd}$ and 4 th antennas are 32,16 and 8 symbols respectively. These subcarrier delays have been used for the simulations reported here. A cyclic delay, $\delta_{c y, l}$, corresponds to the multiplication of a phase factor whose phase is linearly increasing with the subcarrier index $l$, i.e.

$$
\phi_{l}=e^{-j \frac{2 \pi}{N_{F F T}} \delta_{c y, l}}
$$

After cyclic shifting, a cyclic prefix is inserted to avoid intersymbol interference and maintain subcarrier orthogonality over the multipath channel. For each of the transmit antennas, the average energy of the symbols is normalised to be $1 / N_{T}$.

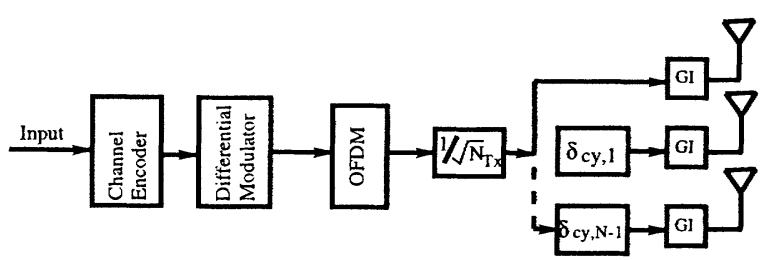

Fig. 2. Differential CDD OFDM Encoder

\section{Receiver}

At the receiver, the arriving signals from each transmit antenna set are summed. The guard interval is then removed and fed to the FFT, as shown in fig. 3. To estimate the transmitted symbols, conventional differential detection is employed [7] as shown by equation 10 , where $\tilde{x}(k)$ is the $k^{t h}$ decoded symbol. After differential demodulation, channel decoding is performed on the symbols using the Viterbi algorithm.

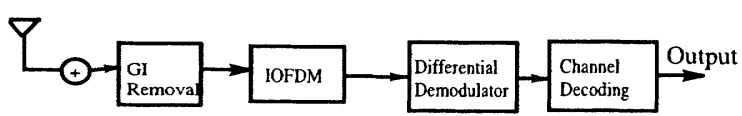

Fig. 3. Differential CDD OFDM Decoder 


$$
\tilde{x}(k)=r(k) r^{*}(k-1)
$$

\section{Channel MOdEL}

A MIMO channel model for $N_{T}$ Tx-antennas and $1 \mathrm{RX}$ antenna is used. The $N_{T} \times 1$ statistically independent channels describe the signal transmission from each TX-antenna $\mathrm{n}, . ., N_{T^{-}}-1$ to the receiver. Each channel has an exponentially decaying delay profile with 8-taps and statistically independent Rayleigh fading characteristic for each tap. The maximum channel delay spread is $\tau_{\max }=400 \mathrm{~ns}$, which is representative of operation in a NLOS indoor environment. The number of impinging rays constituting a single tap is fixed to 12 and a normalised Doppler frequency is assumed to be $f_{D} T_{O F D M}=$ $5 \times 10^{-3}$.

\section{Simulation ARCHITECTURE}

A simulation platform based on the HIPERLAN/2 standard mode 1 [14] has been used to compare the Bit Error Rate (BER) performance between the two transmit diversity schemes. A total bandwidth of $20 \mathrm{MHz}$ with a sampling rate of $0.05 \mu \mathrm{sec}$ has been used. A total of 64 subcarriers giving a useful symbol duration of $3.2 \mu \mathrm{sec}$ is chosen and a mandatory guard interval of $0.8 \mu \mathrm{sec}$ or 16 samples has been employed. A 64 point IFFT/FFT is employed for 64 data subcarriers. This gives a subcarrier spacing of $312.5 \mathrm{kHz}$. A half-rate convolutional encoder (rate $\left.=1 / 2,(133,177)_{8}\right)$ and soft Viterbi decoder is used for channel decoding. The system parameters are summarised in table 2.

TABLE II

SYSTEM PARAMETERS

\begin{tabular}{|c|c|c|}
\hline FFT-length & $N_{F F T}$ & 64 \\
\hline $\begin{array}{l}\text { No. of subcarri- } \\
\text { ers }\end{array}$ & $N_{c}$ & 64 \\
\hline Bandwidth & B & $20 \mathrm{MHz}$ \\
\hline $\begin{array}{l}\text { Subcarrier spac- } \\
\text { ing }\end{array}$ & $\Delta f_{s c}$ & $\mathrm{~B} / N_{\mathrm{c}}=312.5 \mathrm{kHz}$ \\
\hline $\begin{array}{l}\text { OFDM symbol } \\
\text { duration }\end{array}$ & TOFDM & $1 / \Delta f_{s c}=3.2 \mu s e c$ \\
\hline Sampling time & $T_{s}$ & $\begin{array}{l}T_{O F D M} / N_{F F T}= \\
0.05 \mu \text { sec }\end{array}$ \\
\hline $\begin{array}{l}\text { Guard interval } \\
\text { length }\end{array}$ & & $\begin{array}{l}16 \text { samples }=0.8 \\
\mu \text { sec }\end{array}$ \\
\hline Modulation & $M=2$ & DPSK \\
\hline Detection & & Differential decoding \\
\hline Channel Coding & & $\begin{array}{l}(133,171)_{\text {oct }} \text { convo- } \\
\text { lutional code, } \\
\text { rate } 1 / 2 \text {, soft Viterbi } \\
\text { decoding }\end{array}$ \\
\hline Delay profile & & $\begin{array}{l}\text { 8-tap, exponentially } \\
\text { decaying, }\end{array}$ \\
\hline $\begin{array}{l}\text { Max. delay } \\
\text { spread }\end{array}$ & $\tau_{\max }$ & $\tau_{\max }=400 n s$ \\
\hline $\begin{array}{l}\text { Normalised } \\
\text { Doppler } \\
\text { frequency }\end{array}$ & $f_{D} T_{O F D M}$ & $5 \times 10^{-3}$ \\
\hline
\end{tabular}

\section{Simulation Results}

The simulation platform has been used to compare the bit error rate (BER) performance of DCDD and DSTBC OFDM system with an outer channel code. All results are produced as a function of $\left(E_{b} / N_{0}\right)$, defined as the transmitted bit power over the noise power spectral density. The simulated results for all transmit antennas for uncoded DSTBC have been benchmarked accordingly with [8] and [11]. Fig. 4 shows the bit error performance (BER) vs. $E_{b} / N_{0}$ for a $N_{T}=2 \mathrm{DCDD}$ system compared to a $2 \mathrm{Tx}$ antenna system for DSTBC. DCDD with cyclic delay, $\delta_{c y, l}=17$ symbols is chosen. It is shown in the figure that DCDD has a very close performance to DSTBC and only differs by $0.3 \mathrm{~dB}$ at BER of $10^{-3}$ and $10^{-4}$. In figure 5 , the performance of DCDD with $\delta_{c y, l}=32$ symbols shows a $1 \mathrm{~dB}$ degradation as compared to DSTBC at both BER of $10^{-3}$ and $10^{-4}$. In [8] it is described that for BPSK modulation we would require two coefficients for the mapping. These two coefficients will have four different combinations, whereas this is not needed in the DCDD scheme. Complexity of the DCDD receiver is considerably less than that of a DSTBC transmitter.

At the receiver for the DSTBC scheme, detection of the estimated symbols would require four receive symbols with additional complexity. With DCDD, such operation is required only with 2 receive symbols, hence the complexity is reduced and the receiver structure can remain the same. For uncoded DCDD, the performance is equivalent to the uncoded single antenna OFDM systems, as shown in all the figures. A significant gain is observed when coded DCDD is compared with uncoded $D C D D$, which results in a $13 \mathrm{~dB}$ gain at the same BER. However, for DSTBC only a $3 \mathrm{~dB}$ gain is achieved with the same comparison. This shows that, by adding an outer channel code to DCDD OFDM systems has reduced the required $E_{b} / N_{0}$ compared to uncoded DCDD for a given requested BER. And only little decrement occur in DSTBC. The results for uncoded DCDDD have similar performance as in [10], thus shows the reliability of the simulator.

For a $3 \mathrm{Tx}$ antennas and 1Rx antenna coded OFDM system, the results obtained from the simulation are shown in fig. 6 . DCDD with cyclic delay, $\delta_{c y, l}=17,34$ symbols are chosen for the 2nd and 3rd antennas respectively. It is observed that DCDD performance differs from DSTBC by only $1 \mathrm{~dB}$ at high SNR. The same performance difference is obtained when $\delta_{c y, l}$ $=32,16$, as shown in figure 7 . The impact of cyclic delays is therefore not that significant compared to $2 \mathrm{Tx}$ antennas.

Finally, for $4 \mathrm{Tx}$ antennas, DSTBC still outperforms DCDD by an average of $1.2 \mathrm{~dB}$ for both choices of $\delta_{c y, l}$, as shown in the fig. 8 and 9. There is a limit for DCDD performance on the range of cyclic delays that can be used, since little gain is obtained when the cyclic delay is reduced compared to adjacent antennas, as is the case when the number of antennas increases. For all the transmit diversity configurations described above, there is an additional complexity incurred 


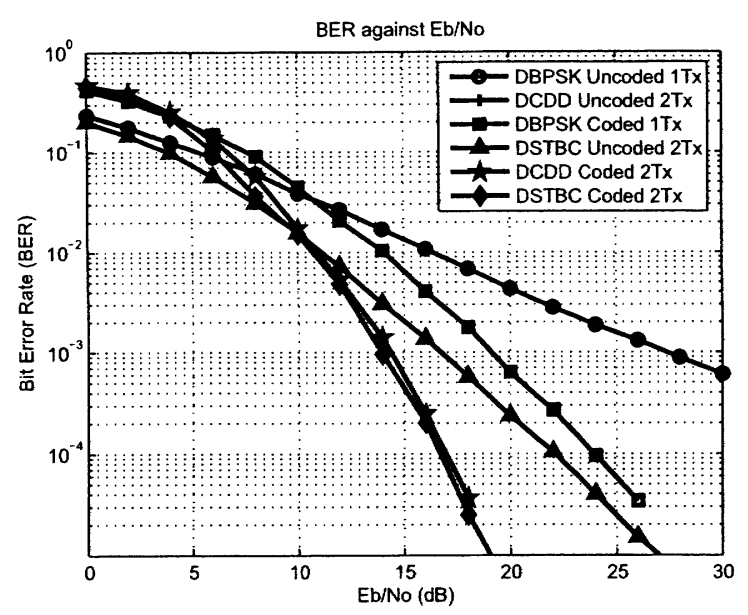

Fig. 4. Differential CDD OFDM and differential STBC OFDM with 2Tx $1 \mathrm{Rx}$ antenna, with cyclic delays on the $2 \mathrm{nd}$ antenna $=17$ symbols

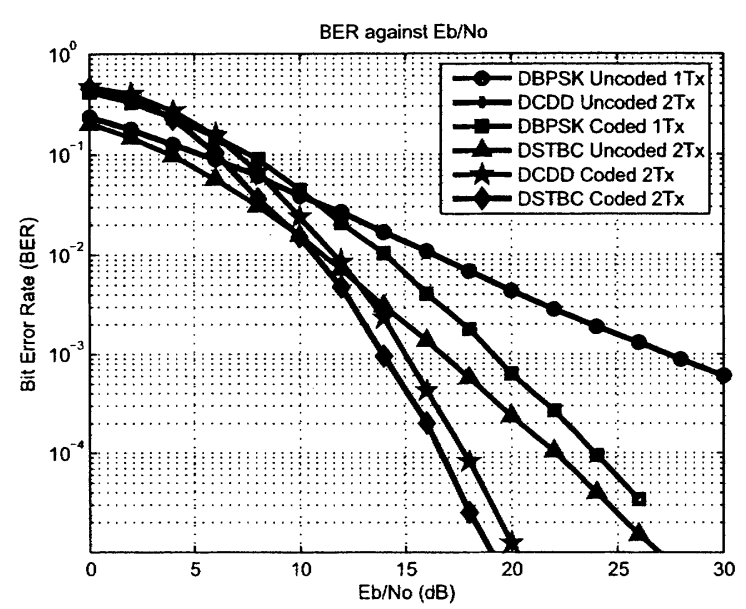

Fig. 5. Differential CDD OFDM and differential STBC OFDM with 2Tx $1 \mathrm{Rx}$ antenna, with cyclic delays on the 2 nd antenna $=32$ symbols

in DSTBC schemes, since the number of inverse fast fourier transforms (IFFTs) is proportional to the number of transmitter required. This transformation is typically $25 \%$ of the transmitter digital baseband complexity [16]. However, this is not the case for DCDD, whereby the operation of cyclic delays is done after the IFFT operation, hence no additional IFFT block is required.

Another significant increase in complexity is the number or multiplication required during the detection of symbols in DSTBC systems. Since, if two symbols are sent with $2 \mathrm{Tx}-$ antennas, there will be 2 multiplication and one addition required. For 3 and $4 \mathrm{Tx}$-antennas, the multiplication is 4 and 3 additional operations. However in DCDD in whatever number of transmit antennas there is always 1 multiplication.

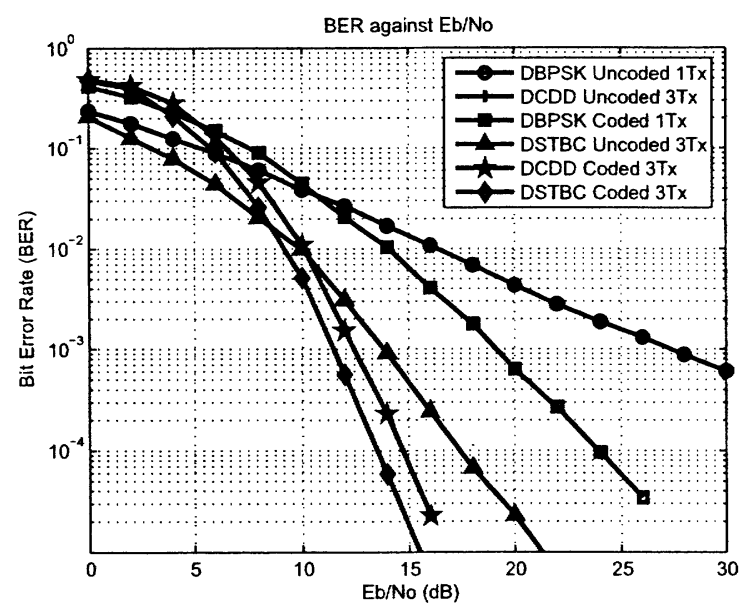

Fig. 6. Differential CDD OFDM and differential STBC OFDM with 3Tx $1 \mathrm{Rx}$ antenna, with cyclic delays on the $2 \mathrm{nd}$ and $3 \mathrm{rd}$ antenna $=17,34$ symbols

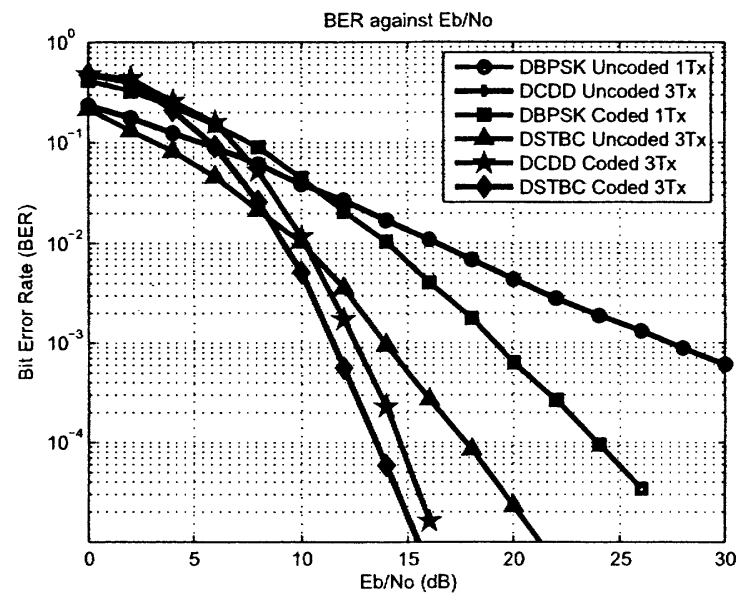

Fig. 7. Differential CDD OFDM and differential STBC OFDM with $3 T x$ $1 \mathrm{Rx}$ antenna, with cyclic delays on the $2 \mathrm{nd}$ and $3 \mathrm{rd}$ antennas $=32,16$ symbols

This shows that the complexity has reduced considerably in DCDD as compared with DSTBC.

\section{CONCLUSIONS}

In this paper we have compared two transmit diversity differential modulation techniques for an OFDM systems. For the DSTBC from orthogonal designs, we have utilised the well known Alamouti scheme and a $3 \mathrm{Tx}$ and $4 \mathrm{Tx}$ rate 1 DSTBC. The competing approach was a standard compatible transmit antenna technique called differential cyclic-delay diversity (DCDD). Simulation results have shown that DSTBC from orthogonal designs outperform DCDD for most of the simulations scenarios by roughly $0.3 \mathrm{~dB}$ for 2 transmit antenna configuration with 17 cyclic delays symbols, the performance has approached very close to DSTBC. For 3 and 4 transmit antennas configurations, DSTBC has gain $1 \mathrm{~dB}$ from DCDD with 


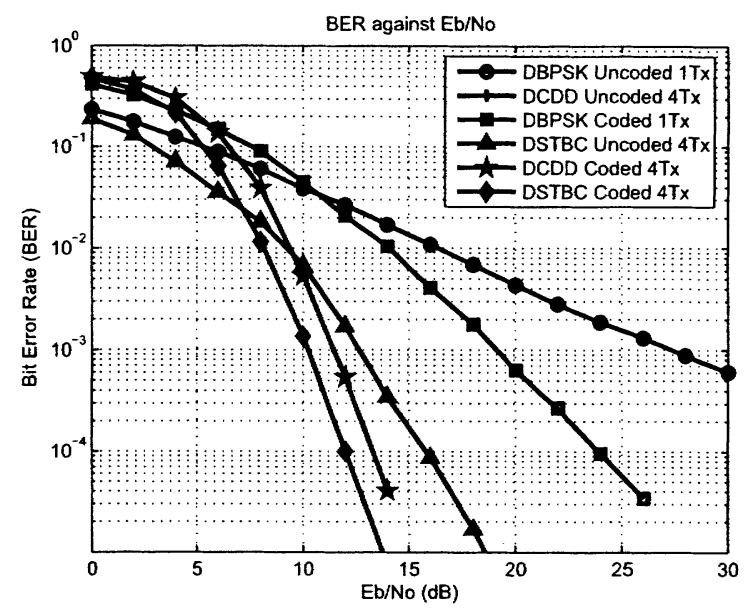

Fig. 8. Differential CDD OFDM and differential STBC OFDM with 4TX $1 \mathrm{Rx}$ antenna, with cyclic delays on the $2 \mathrm{nd}, 3 \mathrm{rd}$ and 4 th antennas $=17,34$, 51 symbols

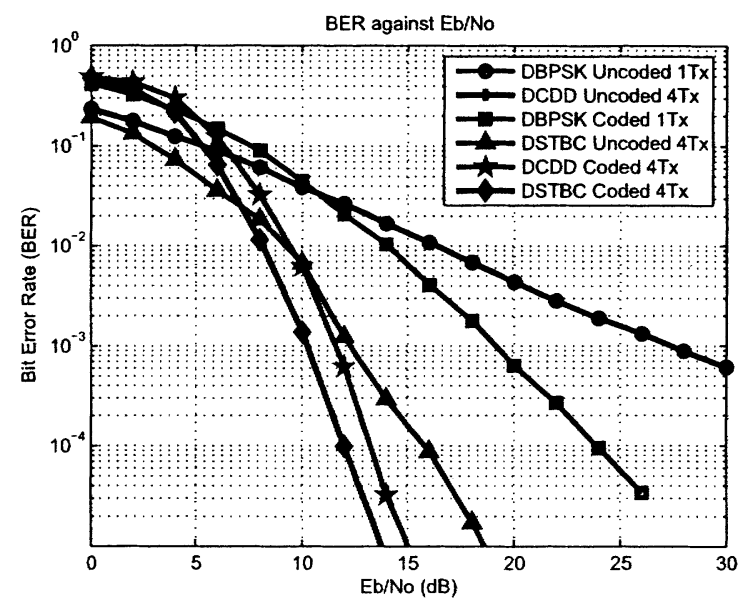

Fig. 9. Differential CDD OFDM and differential STBC OFDM with 4Tx $1 \mathrm{Rx}$ antenna, with cyclic delays on the $2 \mathrm{nd}$, 3rd and 4 th antennas $=32,16$, 8 symbols

cyclic delays chosen. However, there are several advantages of DCDD compared to DSTBC.They are listed as follows:

- There is no change on the receiver side of DCDD scheme, thus is can be compatible with the current standard system.

- The number of Tx-antennas is arbitrary, and no need to design code matrix for specific antenna configurations.

- Low implementation complexity, due to simple cyclic shifts in the time domain.

- Detection at the receiver is simple and no additional multiplication needed in the algorithm.

- No additional IFFT block required in DCDD compared to DSTBC.

\section{REFERENCES}

[1] H. Heiskala and J. Terry, "OFDM Wireless LANs: A Theoretical and Practical Guide", SAMS, 2002.

[2] S.M. Alamouti, "A simple transmit diversity technique for wireless communications", IEEE Journal on Selected Areas in Communications, October 1998, vol.6, no.8, pp. 1451-1458.

[3] V. Tarokh, N. Seshadri and A.R. Calderbank "Space-times codes for high data rates wireless communication: Performance criterion and code construction", IEEE Transactions on Information Theory Communications, March 1998, vol.44, no.2, pp. 744-764.

[4] A. Wittneben, "A New Bandwidth Efficient Transmit Antenna Modulation Diversity Scheme for Linear Digital Modulation," IEEE ICC, May 1993, pp. 1630-34.

[5] A. Dammann and S. Kaiser, "Standard çonformable antenna diversity techniques for OFDM and its application to the DVBT system", In Proceedings IEEE Global Telecommunications Conference on Communication (GLOBECOM 2001), November 2001, pp. 3100-3105.

[6] M. Bossert, A. Huebner, F. Schuehlein, H. Haas and E. Costas, "On Cyclic Delay Diversity in OFDM Based Transmission Schemes", 7th International OFDM-Workshop (InOWo), Hamburg, Germany, September 2002.

[7] J.G. Proakis, "Digital Communications", 3rd Edition, McGraw Hill International Editions, 1995, pp. 274-278.

[8] V. Tarokh and H. Jafarkhani, "A differential detection scheme for transmit diversity ", IEEE Journal on Selected Areas of Communications, July 2000, vol.18, No.7, pp. 1169-1174.

[9] B. Vucetic and J. Yuan, "Space-Time Coding ", John Wiley and Sons, 2003 , pp. 226.

[10] B. Allen, F. Said, G. Bauch, G. Auer, H. Aghvami,"A spectrally efficient transmit diversity scheme for differentially modulated multicarrier transmission ", IEE Proceedings on Communications, June 2004.

[11] H. Jafarkhani and V. Tarokh,"Multiple Transmit Antenna Differential Detection From Generalized Orthogonal Designs ", IEEE Transactions On Information Theory, September 2001, Vol.47, No.6.

[12] A. Huebner, F. Schuehlein, M. Bossert, E. Costa and H. Haas, "A Simple Space-Frequency Coding Scheme with Cyclic Delay Diversity for OFDM ", Sth European Personal Mobile Communications Conference (EPMCC 2003), Glasgow, Scotland, April 2003.

[13] G. Bauch "Aspects of Delay Diversity in OFDM." ICC, May 2005, Seoul, Korea.

[14] European Telecommunications Standard Institute ETSI,"Broadband Radio Access Networks(BRAN); HIPERLAN Type 2; Physical layer ", V1.1.1 (2000-04).

[15] C. Gao, A.M. Haimovich and D. Lao "BER Analysis for Space-Time Block Code with Differential Detection", IEEE Letters, vol: 7, Issue: 7, July 2003 , pp. 314-316.

[16] A. Batra, J. Balakrishnan, G. Roberto, J.R. Foerster and A. Dabak "Design of a Multiband OFDM system for realistic UWB channel environments", IEEE Trans. On Microwave Theory and Techniques, Sept 2004, pp. 2123-2138. 